\title{
Expression and clinical significance of circular RNAs hsa_circ_0000175 and hsa_circ_0008410 in peripheral blood mononuclear cells from patients with rheumatoid arthritis
}

\author{
QING LUO $^{1 *}$, LULU ZENG $^{1 *}$, LINGBING ZENG $^{1}$, JIAYUE RAO $^{2}$, LU ZHANG $^{2}$, \\ YANG GUO $^{1}$, ZIKUN HUANG ${ }^{1}$ and JUNMING LI ${ }^{1}$ \\ ${ }^{1}$ Department of Clinical Laboratory, The First Affiliated Hospital of Nanchang University; \\ ${ }^{2}$ Medical College, Nanchang University, Nanchang, Jiangxi 330006, P.R. China
}

Received July 26, 2019; Accepted December 30, 2019

DOI: $10.3892 /$ ijmm.2020.4498

\begin{abstract}
Circular RNAs (circRNAs) are a novel class of RNAs that may be used as biomarkers in clinical blood samples. However, the role of circRNAs in rheumatoid arthritis (RA) has not been extensively investigated. In the present study, six circRNAs, including hsa_circ_0082689, hsa_circ_0087798,hsa_circ_0000175, hsa_circ_0008410, hsa_circ_0049356 and hsa_circ_0032959 levels were determined in peripheral blood mononuclear cells (PBMCs) collected from 24 patients with RA and 24 healthy controls (HC) by reverse transcription-quantitative polymerase chain reaction (RT-qPCR) analysis. Hsa_circ_0000175 and hsa_circ_0008410 were selected for further evaluation in an independent cohort consisting of 63 patients with RA, 50 with systemic lupus erythematosus (SLE), 24 with ankylosing spondylitis (AS) and $21 \mathrm{HC}$. Spearman's rank correlation coefficient was used to analyze the correlation between these two circRNAs and the clinical characteristics of RA, and receiver operating characteristic (ROC) curves were constructed to evaluate their value in RA diagnosis. Multivariate analysis (logistic regression) was used to analyze the risk factors. Of the six selected circRNAs, the expression of hsa_circ_0000175 was found to be significantly reduced and the expression of hsa_circ_0008410 was significantly elevated in PBMCs from patients with RA compared with their levels in HC. The expression of hsa_circ_0000175 in patients with RA was correlated with anti-citrullinated protein antibodies, white blood cell
\end{abstract}

Correspondence to: Dr Junming Li or Dr Zikun Huang, Department of Clinical Laboratory, The First Affiliated Hospital of Nanchang University, 17 Yongwai Zheng Jie, Nanchang, Jiangxi 330006, P.R. China

E-mail: lisir361@163.com

E-mail: yfyhzk@163.com

*Contributed equally

Key words: biomarker, circular RNAs, rheumatoid arthritis count, lymphocyte count, lymphocyte percentage, neutrophil count, neutrophil percentage and neutrophil-to-lymphocyte ratio. Furthermore, the expression of hsa_circ_0008410 was correlated with tender joint count, disease duration, platelet count and plateletcrit, indicating the activity and severity of RA. ROC curve analysis suggested that hsa_circ_0000175, hsa_circ_0008410, and the combination of hsa_circ_0000175 and hsa_circ_0008410 have significant value in the diagnosis of RA. Hsa_circ_0000175 and hsa_circ_0008410 also differed significantly between patients with RA, and those with SLE and AS. Moreover, logistic regression analysis revealed that the expression of PBMC hsa_circ_0000175 and hsa_circ_0008410 were risk factors for RA. Therefore, PBMC hsa_circ_0000175,hsa_circ_0008410, and the combination of PBMC hsa_circ_0000175 and hsa_circ_0008410 may improve the diagnostic accuracy for RA. In addition, the expression levels of PBMC hsa_circ_0000175 and hsa_circ_0008410 were associated with disease activity and severity of RA.

\section{Introduction}

Rheumatoid arthritis (RA) is a chronic debilitating systemic autoimmune disease that affects $1 \%$ of the population and may lead to permanent joint destruction resulting insignificant morbidity (1). Early and accurate diagnosis is important for selecting an effective treatment, maintaining the quality of life and improving the survival rate of RA. The current conventional methods for RA diagnosis primarily include the American College of Rheumatology (ACR) classification criteria (2). However, The ACR RA classification criteria, including clinical and laboratory characteristics, have various disadvantages regarding early diagnosis (3), such as subjective clinical diagnosis, the need for refinement using objective and more sensitive imaging modalities, and the relatively low sensitivity of anti-citrullinated protein antibodies (ACPA) for RA (72\%). Therefore, identifying appropriate novel biomarkers for early diagnosis of RA is urgently required.

Novel diagnostic methods, such as linear RNAs, including microRNAs (miRNAs) and long non-coding RNAs, have been demonstrated to be useful as biomarkers for the diagnosis of RA (4-7). Circular RNAs (circRNAs) are a novel class of RNAs 
that form from the covalent linkage of the $3^{\prime}$ and $5^{\prime}$ ends to produce a closed loop $(8,9)$. Accumulating evidence indicates that several circRNAs regulate various physiological and pathological processes by acting as competing endogenous RNAs (ceRNAs) to restrain the activity of miRNAs (10-12). circRNAs differ from linear RNAs in that they can resist RNase digestion and exhibit high stability, which allows circRNAs to be selectively enriched during sample processing and makes them more suitable biomarkers compared with linear RNAs $(13,14)$. Recent evidence has indicated that circRNAs may serve as novel biomarkers in the diagnosis and prognosis of numerous diseases $(15,16)$. In 2017, Ouyang et al (17) confirmed that five circRNAs (092516, 003524, 103047, 104871 and 101873) that were found to be increased in peripheral blood mononuclear cells (PBMCs) from patients with RA are potential biomarkers for RA diagnosis. Our previous 2018 study revealed that peripheral blood hsa_circ_0044235 may act as a biomarker for RA diagnosis (18). More recently, Tang et al (19) quantified the elevated expression of ciRS-7 in PBMC and suggested that it may be a suitable diagnostic biomarker for RA. However, the diagnostic value of these reported circRNAs is not optimal, due to the low or moderate area under the receiver operating characteristic (ROC) curve (AUC). Therefore, novel circRNA biomarkers for early diagnosis and prognosis of RA must be identified. Our previous study revealed that certain circRNAs in PBMCs were differentially expressed between patients with systemic lupus erythematosus (SLE), which is a common autoimmune disease, and healthy controls (HC) (20). Considering that RA is also a common autoimmune disease, three upregulated (hsa_circ_0082689, hsa_circ_0087798, hsa_ circ_0000175) and three downregulated (hsa_circ_0008410, hsa_circ_0049356 and hsa_circ_0032959) circRNAs, which were found to be significantly aberrant in both peripheral blood mononuclear cells (PBMCs) from patients with SLE in our previous study (20), and T cells from patients with SLE in previous literature (21), were selected to explore the possibility of being used as biomarkers for diagnosis of RA.

\section{Materials and methods}

Patient variables. Patients $(\mathrm{n}=87)$ who fulfilled the revised ACR 2010 criteria for RA (2) were consecutively enrolled from The First Affiliated Hospital of Nanchang University (Jiangxi, China) between September 2018 and March 2019. Those RA patients accompanied by other autoimmune, inflammatory or hormonal disease, cancer or mental disorder were excluded. All patients had new-onset RA and had not received corticosteroids or immunosuppressive drugs prior to recruitment. Then, 9 new-onset RA cases were administered therapeutic regimens with corticosteroids and immunosuppressive drugs for at least 15 days. The information on disease activity score 28 (DAS28), swollen joint count (SJC), tender joint count (TJC), patient visual analogue scale (VAS), disease activity, erythrocyte sedimentation rate (ESR), C-reactive protein (CRP), ACPA, rheumatoid factor (RF), white blood cell count (WBC), red blood cell count (RBC), hemoglobin, hematocrit (HCT), platelet count (PLT), mean platelet volume (MPV), plateletcrit (PCT), platelet distribution width (PDW), lymphocyte count (L), lymphocyte percentage (L\%), monocyte count $(\mathrm{M})$, monocyte percentage $(\mathrm{M} \%)$, neutrophil count $(\mathrm{N})$, neutrophil percentage $(\mathrm{N} \%)$, neutrophil-to-lymphocyte ratio (NLR) and platelet-to-lymphocyte ratio (PLR) was collected. $\mathrm{HC}(\mathrm{n}=45)$ without autoimmune or inflammatory diseases and who were also unrelated to patients with autoimmune diseases were randomly enrolled from the First Affiliated Hospital of Nanchang University between September 2018 and March 2019. As a disease control, 91 patients with autoimmune diseases [50 SLE and 41 ankylosing spondylitis (AS) cases] clinically confirmed by the diagnostic criteria of SLE (22) and the diagnostic criteria of AS (23) after excluding RA, were also enrolled from the First Affiliated Hospital of Nanchang University during the same period. All study protocols complied with the principles outlined in the Declaration of Helsinki and were approved by the Ethics Committee of the First Affiliated Hospital of Nanchang University (ethics no. 019). All participants provided signed informed consent.

Collection of PBMCs and total RNA extraction. Blood samples ( $2 \mathrm{ml}$ ) were collected from all subjects in K2-EDTA tubes, and PBMCs were isolated by density-gradient centrifugation using Ficoll-Paque Plus (GE Healthcare Life Sciences) at $25^{\circ} \mathrm{C}$. TRIzol ${ }^{\circledR}$ reagent (Invitrogen; Thermo Fisher Scientific, Inc.) was added to the PBMCs and stored at $-80^{\circ} \mathrm{C}$. Total RNA extraction from PBMC specimens was carried out according to the manufacturer's instructions. The concentration and integrity of the RNA was assessed by a NanoDrop ND-1000 spectrophotometer (Agilent Technologies, Inc.).

Reverse transcription-quantitative PCR (RT-qPCR) analysis. RT and qPCR were performed with PrimeScript ${ }^{\mathrm{TM}}$ RT kit (Takara Bio Inc.) and SYBR Premix Ex Taq ${ }^{\mathrm{TM}}$ II (Takara Bio Inc.), respectively. RT-qPCR was performed on an ABI 7500 Real Time PCR system (Applied Biosystems; Thermo Fisher Scientific, Inc.) with the following PCR thermocycler protocol: Initial denaturation step at $95^{\circ} \mathrm{C}$ for $5 \mathrm{~min}$, followed by 40 cycles of $95^{\circ} \mathrm{C}$ for $15 \mathrm{sec}$ (denaturation), $60^{\circ} \mathrm{C}$ for $1 \mathrm{~min}$ (annealing and elongation) and $72^{\circ} \mathrm{C}$ for $2 \mathrm{~min}$ (final extension). The selected circRNAs for RT-qPCR analysis are shown in Table I. $\beta$-actin was used as an internal control. The primers used in RT-qPCR are listed in Table II. The data were analyzed using the $2^{-\Delta \Delta \mathrm{Cq}}$ method (15).

Statistical analysis. Statistical analysis and graphic presentation were carried out with GraphPad Prism 5.0 (GraphPad Software, Inc.) and SPSS version 17.0 (SPSS Inc.). The equation $n=Z^{2} \times \sigma^{2} / d^{2}=1.28^{2} \times 0.5^{2} / 0.1^{2}=40.96$ was used to calculate minimal sample size. A Student's t-test was used between two groups where the samples passed the normality test; otherwise, the non-parametric Mann-Whitney test was used to analyze the data. The paired t-test was performed for evaluation of changes in treatment. Kruskal-Wallis test was used for statistical analysis between three or more groups followed by a Dunn's post-hoc test for subsequent analyses between individual groups. Spearman's rho method was used for correlation analysis. Multivariate analysis (logistic regression) was used to analyze the risk factors. ROC curves were constructed to evaluate the diagnostic value of circRNAs that were dysregulated in the PBMCs of patients with RA compared with HC, SLE and AS. $\mathrm{P}<0.05$ was considered to indicate statistically significant differences. 
Table I. Details of the selected circRNAs.

\begin{tabular}{llllll}
\hline circRNAs & Source & Chrom & Strand & circRNA_type & GeneSymbol \\
\hline hsa_circ_0082689 & circBase & chr7 & - & Exonic & PARP12 \\
hsa_circ_0087798 & circBase & chr9 & + & Exonic & NIPSNAP3A \\
hsa_circ_0000175 & circBase & chr1 & - & Exonic & ELK4 \\
hsa_circ_0008410 & circBase & chr17 & + & Intronic & PGS1 \\
hsa_circ_0049356 & circBase & chr19 & + & Exonic & CARM1 \\
hsa_circ_0032959 & circBase & chr14 & - & Exonic & CCDC88 \\
\hline
\end{tabular}

circRNAs, circular RNAs.

Table II. Specific circRNAs primers used for RT-qPCR analysis.

$\begin{array}{lll}\text { circRNAs } & \text { Primer sequence }(F) & \text { Primer sequence }(\mathrm{R})\end{array}$

\begin{tabular}{ll}
\hline hsa_circ_0082689 & GTCCCCAAACACTCTTAGCCA \\
hsa_circ_0087798 & GCAGTTCATGTTCTTTGGTGGA \\
hsa_circ_0000175 & GCCCATTTTCCCCAGACCTAC \\
hsa_circ_0008410 & CTGCTTTTGTCCTTGAAGCCAG \\
hsa_circ_0049356 & CACCAAGGCCAACTTCTGGTA \\
hsa_circ_0032959 & ACAGCTGGACATTGAGACCC \\
$\beta$-actin & TACTGCCCTGGCTCCTAGCA
\end{tabular}

CACACTCAGGTTGTGTTCGG
CTGGGTCCCGTAGCAAAAGA
GGAACTGCCACAGGGTGATA
CACCAGCTCCGTGAAGAAGTC
CGGTCCGTCAGGTTGTTACT
TTTCCTCTCACACTGGACAGC
TGGACAGTGAGGCCAGGATAG

circRNAs, circular RNAs; RT-qPCR, reverse transcription-quantitative polymerase chain reaction; F, forward; R, reverse.

\section{Results}

Characteristics of the study subjects. A total of 223 subjects were enrolled in the present study, including 87 patients with RA, 50 patients with SLE, $45 \mathrm{HC}$ and 41 patients with AS. RA patients were classified into screening and validation cohorts. The screening cohort included 24 RA patients and $24 \mathrm{HC}$. An independent cohort consisting of $63 \mathrm{RA}$ patients and $21 \mathrm{HC}$ were enrolled in the validation set for evaluation of abnormal circRNAs. The characteristics of the study subjects are summarized in Table III. There were no significant differences between RA patients and HC regarding age or sex. Due to the differences in age and sex among RA, AS and SLE patients (the incidence of RA was high among women of 50-60 years, the incidence of AS was high among young male patients and the incidence of SLE was high among women of childbearing age), patients with RA, AS and SLE were not age-matched, and patients with RA and AS were not sex-matched in the present study. No correlation between circRNA levels and age or sex was observed in AS, SLE, RA or HC (data not shown).

PBMC hsa_circ_0000175 and hsa_circ_0008410 expression is abnormal in RA patients. The expression of hsa_circ_0082689, hsa_circ_0087798, hsa_circ_0000175, hsa_circ_0008410,hsa_circ_0049356 and hsa_circ_0032959 in PBMCs was first detected in the screening cohort including 24 RA patients and $24 \mathrm{HC}$ using RT-PCR. Compared with HC, the expression of hsa_circ_0000175 and hsa_circ_0008410 was significantly different in the PBMCs from patients with RA (all $\mathrm{P}<0.05$, Fig. 1), while the expression of hsa_circ_0082689, hsa_circ_0087798,hsa_circ_0049356 and hsa_circ_0032959 did not significantly differ between the two groups.

Validation of PBMC hsa_circ_0000175 and hsa_ circ_0008410 expression in the second stage. Subsequently, the expression of hsa_circ_0000175 and hsa_circ_0008410 in PBMCs was verified in an independent cohort, including $63 \mathrm{RA}$ patients and $21 \mathrm{HC}$. In accordance with the screening results, the expression of hsa_circ_0000175 in PBMCs from 63 RA patients was significantly decreased compared with that in $21 \mathrm{HC}(\mathrm{P}<0.0001$, Fig. $2 \mathrm{~A})$, whereas the expression of hsa_circ_0008410 was significantly increased in the PBMCs from 63 RA patients compared with that in $21 \mathrm{HC}(\mathrm{P}<0.0001$, Fig. 2B). When considering the data from all $87 \mathrm{RA}$ patients and $45 \mathrm{HC}$, the same patterns were observed in hsa_circ_0000175 and hsa_circ_0008410 between RA patients and HC (both $\mathrm{P}<0.0001$, Fig. 2C and D).

Correlation of PBMC hsa_circ_0000175 and hsa_ circ_0008410 expression with clinical characteristics of $R A$. To investigate whether the expressions of PBMC hsa circ_0000175 and hsa_circ_0008410 in RA patients could be considered as biomarkers for the activity and severity of the disease, Spearman's rho method was used to assess the association between the expression of PBMC hsa_circ_0000175 and hsa_circ_0008410 in RA patients with clinical characteristics, including DAS28, TJC, TJC, VAS, disease duration, ACPA, RF, ESR, CRP, WBC, RBC, HGB, HCT, PLT, MPV, 
Table III. Clinical characteristics of the patients with RA, HC, SLE and AS.

\begin{tabular}{|c|c|c|c|c|}
\hline Clinical characteristic & RA & $\mathrm{HC}$ & AS & SLE \\
\hline Number of subjects & 87 & 45 & 41 & 50 \\
\hline \multicolumn{5}{|l|}{ Sex } \\
\hline Male & 16 & 10 & $29^{\mathrm{b}}$ & 4 \\
\hline Female & 71 & 35 & $12^{\mathrm{b}}$ & 46 \\
\hline Age, years & $49.89 \pm 12.86$ & $45.24 \pm 13.43$ & $31.02 \pm 9.96^{\mathrm{b}}$ & $35.85 \pm 15.48^{c}$ \\
\hline Days since diagnosis & $1,400.01 \pm 2,268.53$ & & & \\
\hline DAS28-ESR & $5.99 \pm 1.51$ & & & \\
\hline DAS28-CRP & $5.35 \pm 1.38$ & & & \\
\hline SJC & $10.83 \pm 7.65$ & & & \\
\hline PJC & $12.53 \pm 7.54$ & & & \\
\hline VAS & $48.44 \pm 31.39$ & & & \\
\hline $\mathrm{RF}, \mathrm{IU} / \mathrm{ml}$ & $436.02 \pm 558.63$ & & & \\
\hline ACPA, RU/ml & $911.04 \pm 972.24$ & & & \\
\hline $\mathrm{ESR}, \mathrm{mm} / \mathrm{h}$ & $51.04 \pm 33.32$ & & $20.08 \pm 20.85^{\mathrm{b}}$ & $63.49 \pm 36.74$ \\
\hline CRP, mg/l & $27.54 \pm 32.13$ & & $11.08 \pm 16.04^{\mathrm{b}}$ & $17.86 \pm 33.18$ \\
\hline WBC, $10^{9} / 1$ & $7.97 \pm 2.39^{\mathrm{a}}$ & $5.74 \pm 0.83$ & $7.40 \pm 1.66$ & $6.32 \pm 3.66^{c}$ \\
\hline $\mathrm{RBC}, 10^{12} / 1$ & $4.37 \pm 0.51^{\mathrm{a}}$ & $4.56 \pm 0.37$ & $4.78 \pm 0.71^{b}$ & $3.75 \pm 0.82^{c}$ \\
\hline HGB, g/l & $124.60 \pm 19.99^{\mathrm{a}}$ & $138.87 \pm 11.37$ & $140.78 \pm 20.59^{b}$ & $117.66 \pm 77.67^{\mathrm{c}}$ \\
\hline HCT, $1 / 1$ & $0.38 \pm 0.05^{\mathrm{a}}$ & $0.41 \pm 0.03$ & $0.43 \pm 0.06^{\mathrm{b}}$ & $0.33 \pm 0.08^{c}$ \\
\hline PLT, $10^{9} / 1$ & $329.36 \pm 121.63^{a}$ & $244.20 \pm 51.96$ & $319.88 \pm 69.42$ & $201.50 \pm 105.79^{c}$ \\
\hline MPV, fl & $10.22 \pm 1.41^{\mathrm{a}}$ & $10.91 \pm 0.89$ & $9.46 \pm 1.02^{b}$ & $10.63 \pm 1.27$ \\
\hline PCT, \% & $0.33 \pm 0.11^{\mathrm{a}}$ & $0.26 \pm 0.05$ & $0.30 \pm 0.06$ & $0.25 \pm 0.09^{c}$ \\
\hline PDW, fl & $12.61 \pm 3.29^{a}$ & $12.9 \pm 2.48$ & $14.06 \pm 2.62^{\mathrm{b}}$ & $12.95 \pm 2.67$ \\
\hline $\mathrm{L}, 10^{9} / 1$ & $1.64 \pm 0.58^{\mathrm{a}}$ & $1.84 \pm 0.31$ & $2.08 \pm 0.57^{b}$ & $1.25 \pm 0.64^{c}$ \\
\hline $\mathrm{L}, \%$ & $21.57 \pm 8.51^{\mathrm{a}}$ & $32.41 \pm 5.31$ & $28.51 \pm 7.13^{\mathrm{b}}$ & $23.37 \pm 10.46$ \\
\hline $\mathrm{M}, 10^{9} / 1$ & $0.43 \pm 0.17^{\mathrm{a}}$ & $0.35 \pm 0.07$ & $0.44 \pm 0.15$ & $0.49 \pm 0.39$ \\
\hline $\mathrm{M}, \%$ & $5.63 \pm 2.04^{\mathrm{a}}$ & $6.12 \pm 1.30$ & $6.07 \pm 1.96$ & $7.77 \pm 3.24^{\mathrm{c}}$ \\
\hline $\mathrm{N}, 10^{9} / 1$ & $5.74 \pm 2.25^{\mathrm{a}}$ & $3.42 \pm 0.71$ & $4.70 \pm 1.38^{\mathrm{b}}$ & $4.51 \pm 3.16^{\mathrm{c}}$ \\
\hline $\mathrm{N}, \%$ & $70.47 \pm 10.45^{\mathrm{a}}$ & $59.04 \pm 5.95$ & $62.98 \pm 7.53^{\mathrm{b}}$ & $67.80 \pm 11.87$ \\
\hline PLR & $237.62 \pm 181.47^{\mathrm{a}}$ & $136.03 \pm 36.19$ & $164.94 \pm 57.32^{\mathrm{b}}$ & $191.23 \pm 136.05^{\mathrm{c}}$ \\
\hline NLR & $4.12 \pm 2.73^{a}$ & $1.90 \pm 0.50$ & $2.43 \pm 0.98^{b}$ & $4.26 \pm 3.84$ \\
\hline
\end{tabular}

${ }^{\mathrm{a}} \mathrm{P}<0.05 \mathrm{RA}$ compared to $\mathrm{HC},{ }^{\mathrm{b}} \mathrm{P}<0.05$ AS compared to RA, ${ }^{\mathrm{C}} \mathrm{P}<0.05$ SLE compared to RA. ACPA, anticitrullinated protein antibodies; AS, ankylosing spondylitis; CRP, C-reactive protein; DAS28, disease activity score; ESR, erythrocyte sedimentation rate; HC, healthy controls; HCT, hematocrit; HGB, hemoglobin; L, lymphocyte count; L\%, lymphocyte percentage; M, monocyte count; M\%, monocyte percentage; MPV, mean platelet volume; N, neutrophils count; N\%, neutrophils percentage; NLR, neutrophil-to-lymphocyte ratio; PCT, plateletcrit; PDW, platelet distribution width; PLR, platelet-to-lymphocyte ratio; PLT, platelet count; PJC, pain joint count; RA, Rheumatoid arthritis; RBC, red blood cell count; RF, rheumatoid factors; SLE, systemic lupus erythematosus; SJC, swollen joint count; VAS, visual analogue scale; WBC, white blood cell count.

PCT, PDW, L, L\%, M, M\%, N, N\%, NLR and PLR. As shown in Fig. 3, the expression of PBMC hsa_circ_0000175 in RA patients was correlated with ACPA $\left(\mathrm{r}_{\mathrm{s}}=-0.294, \mathrm{P}=0.0090\right)$, WBC $\left(r_{s}=-0.246, P=0.0216\right), L\left(r_{s}=0.226, P=0.0356\right), L \%$ $\left(r_{\mathrm{s}}=0.350, \mathrm{P}=0.0009\right), \mathrm{N}\left(\mathrm{r}_{\mathrm{s}}=-0.295, \mathrm{P}=0.0056\right), \mathrm{N} \%\left(\mathrm{r}_{\mathrm{s}}=-0.343\right.$, $\mathrm{P}=0.0011)$ and NLR $\left(\mathrm{r}_{\mathrm{s}}=-0.367, \mathrm{P}=0.0005\right)$, and the expression of PBMC hsa_circ_0008410 in RA patients was correlated with TJC $\left(r_{s}=0.213, P=0.0471\right)$, disease duration $\left(r_{s}=0.211\right.$, $\mathrm{P}=0.0498)$, PLT $\left(\mathrm{r}_{\mathrm{s}}=0.241, \mathrm{P}=0.0247\right)$ and PCT $\left(\mathrm{r}_{\mathrm{s}}=0.267\right.$, $\mathrm{P}=0.0138$ ), which indicated the activity and severity of RA.

Subsequently, the expression of PBMC hsa_circ_0000175 and hsa_circ_0008410 was detected in 9 new-onset RA cases pre- and post-treatment; however, there was no difference between pre- and post-treatment levels (data not shown).

Diagnostic value of PBMC hsa_circ_0000175 and hsa_ circ_0008410 expression in RA patients. Next, ROC curves were produced to investigate the diagnostic value of PBMC hsa_circ_0000175 and hsa_circ_0008410 expression in RA. The data indicated that PBMC hsa_circ_0000175 expression had a moderate ability to distinguish RA patients from $\mathrm{HC}$, with an AUC of 0.835 , a cut-off of $<0.936$, a sensitivity of $86.21 \%$ and a specificity of $73.33 \%$ (Fig. 4A), PBMC hsa_circ_0008410 expression also had a moderate ability to 
A

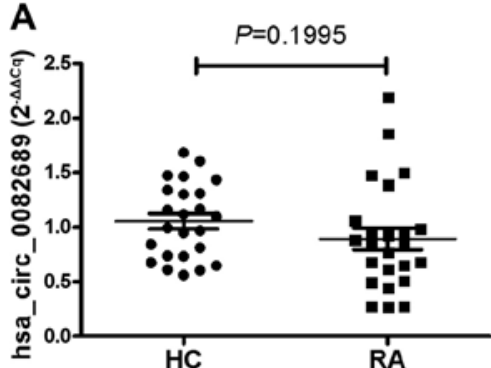

D

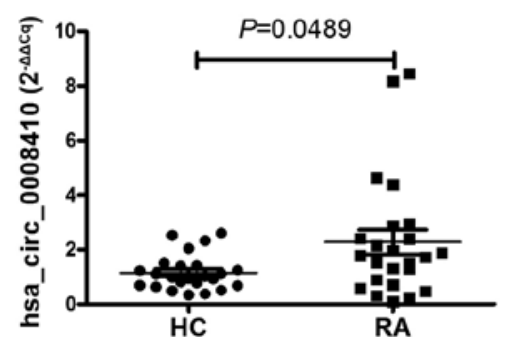

B

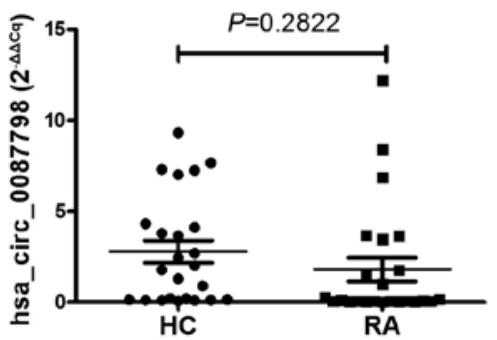

E

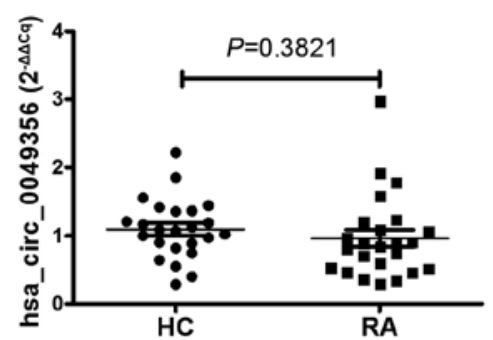

C

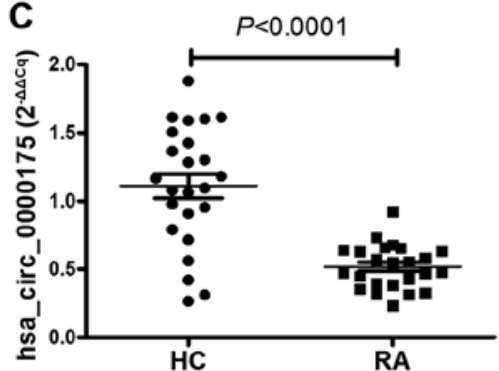

$\mathbf{F}$

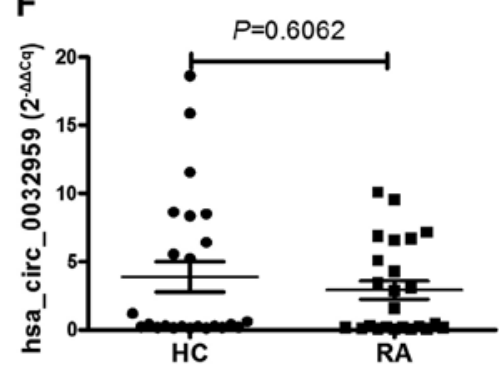

Figure 1. Screening of abnormally expressed circRNAs in PBMCs from 24 RA patients and 24 HC. (A) The expression of hsa_circ_0082689 exhibited no differences between the two groups (Student's t-test). (B) The expression of hsa_circ_0087798 exhibited no differences between the two groups (Student's t-test). (C) The expression of hsa_circ_0000175 in patients with RA was significantly lower compared with that in HC (Mann-Whitney U test). (D) The expression of hsa_circ_0008410 in patients with RA was significantly higher compared with that in HC (Mann-Whitney U test). (E) The expression of hsa_circ_0049356 exhibited no differences between the two groups (Student's t-test). (F) The expression of hsa_circ_0032959 exhibited no differences between the two groups (Mann-Whitney test). CircRNAs, circular RNAs; HC, healthy controls; PBMC, peripheral blood mononuclear cells; RA, rheumatoid arthritis.

A

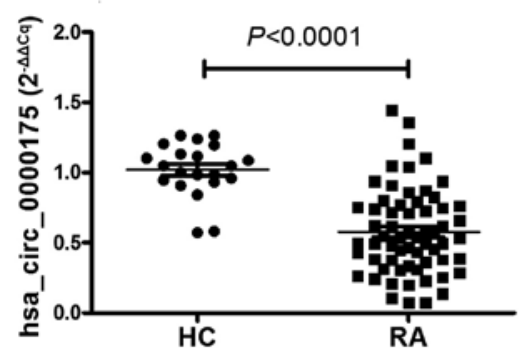

C

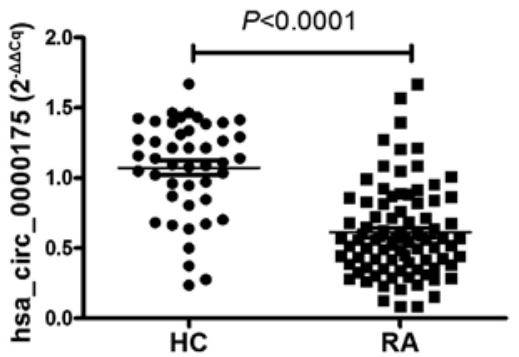

B

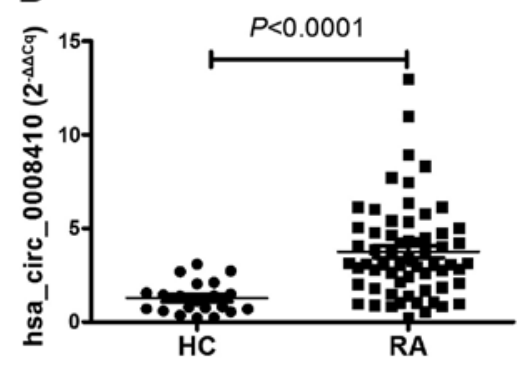

D

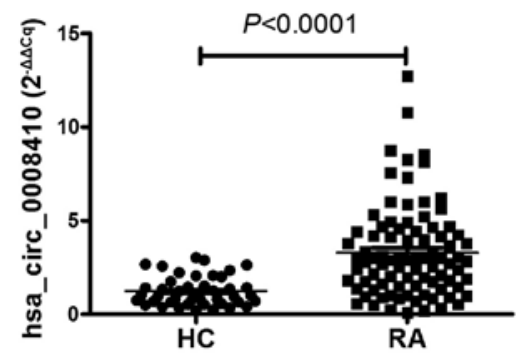

Figure 2. Validation the expression of PBMC hsa_circ_0000175 and hsa_circ_0008410 in the second stage. (A) The expression of hsa_circ_0000175 in PBMCs from 63 RA patients was significantly decreased compared with that in 21 HC (Mann-Whitney test). (B) The expression of hsa_circ_0008410 in PBMCs from 63 RA patients was significantly increased compared with that in 21 HC (Mann-Whitney test). (C) The expression of hsa_circ_0000175 in PBMC from all $87 \mathrm{RA}$ patients was significantly decreased compared with that in all $45 \mathrm{HC}$ (Student's t-test). (D) The expression of hsa_circ 0008410 in PBMC from all $87 \mathrm{RA}$ patients was significantly increased compared with that in all $45 \mathrm{HC}$ (Mann-Whitney test). HC, healthy controls; PBMC, peripheral blood mononuclear cells; RA, rheumatoid arthritis.

distinguish RA patients from HC, with an AUC of 0.804, a cut-off of $>2.685$, a sensitivity of $55.17 \%$ and a specificity of $95.56 \%$ (Fig. 4B). Moreover, the logistic regression model revealed that the combination of PBMC hsa_circ_0000175 and hsa_circ_0008410 expression exhibited an improved ability to distinguish RA patients from HC, with an AUC of 0.971, a sensitivity of $93.10 \%$ and a specificity of $93.33 \%$ (Fig. 4C). These results demonstrated that the combination of PBMC hsa_circ_0000175 and hsa_circ_0008410 expression may be a useful biomarker in RA. 
A
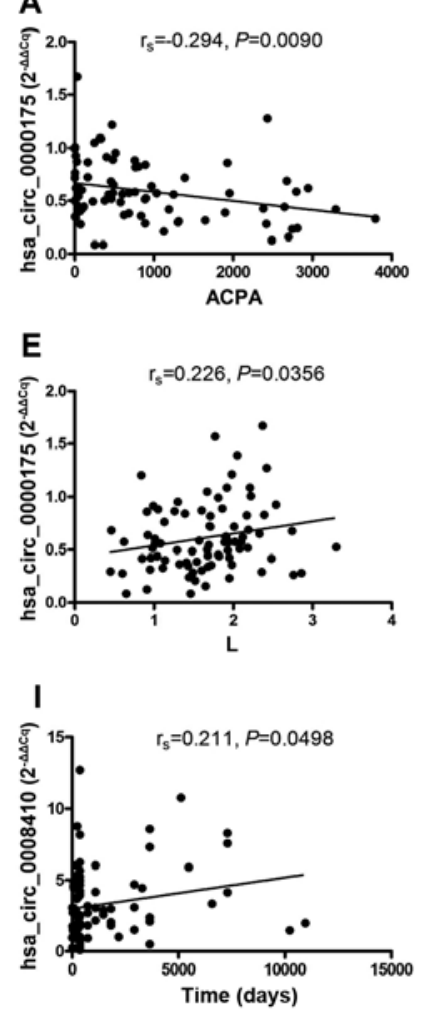

B
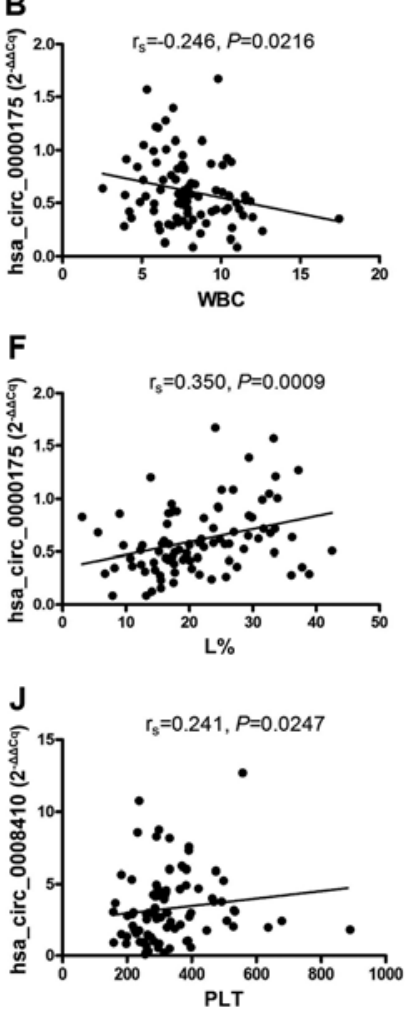
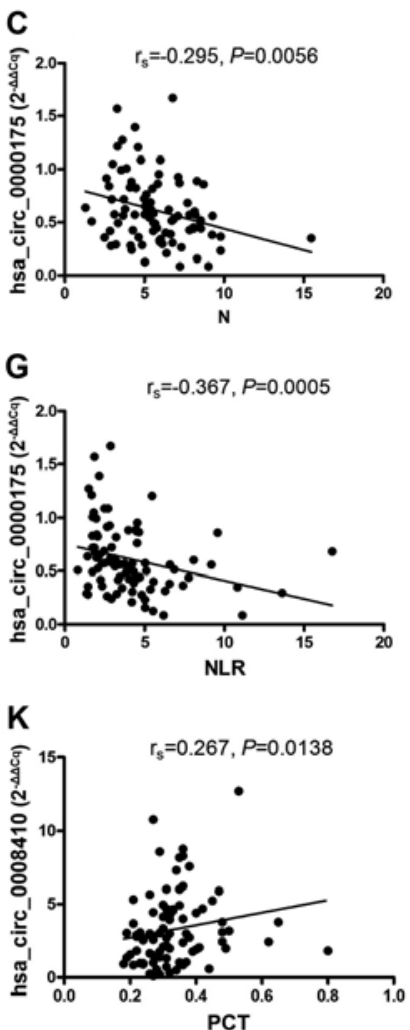
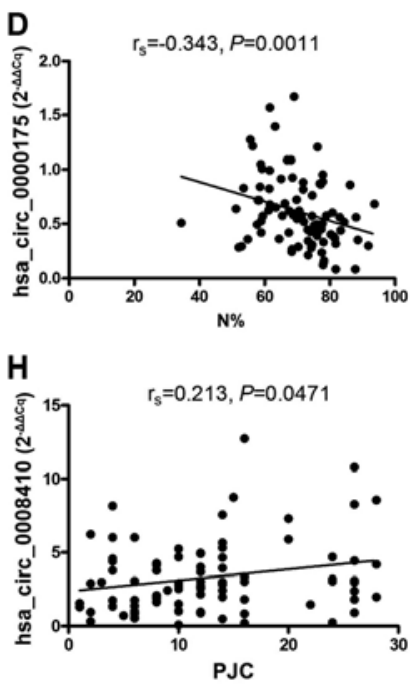

Figure 3. Correlation of PBMC hsa_circ_0000175 and hsa_circ_0008410 expression with clinical characteristics of RA. (A) The expression of PBMC hsa_circ_0000175 in RA patients was negatively correlated with ACPA (Spearman's method). (B) The expression of PBMC hsa_circ_0000175 in RA patients was negatively correlated with WBC (Spearman's method). (C) The expression of PBMC hsa_circ_0000175 in RA patients was negatively correlated with N (Spearman's method). (D) The expression of PBMC hsa_circ_0000175 in RA patients was negatively correlated with N\% (Spearman's method). (E) The expression of PBMC hsa_circ_0000175 in RA patients was positively correlated with L (Spearman's method). (F) The expression of PBMC hsa_circ_0000175 in RA patients was positively correlated with L\% (Spearman's method). (G) The expression of PBMC hsa_circ_0000175 in RA patients was negatively correlated with NLR (Spearman's method). (H) The expression of PBMC hsa_circ_0008410 in RA patients was positively correlated with TJC (Spearman's method). (I) The expression of PBMC hsa_circ_0008410 in RA patients was positively correlated with disease duration (Spearman's method). (J) The expression of PBMC hsa_circ_0008410 in RA patients was positively correlated with PLT (Spearman's method). (K) The expression of PBMC hsa_circ_0008410 in RA patients was positively correlated with PCT (Spearman's method). ACPA, anti-citrullinated protein antibodies; L, lymphocyte count; L\%, lymphocyte percentage; N, neutrophil count; N\%, neutrophil percentage; NLR, neutrophil-to-lymphocyte ratio; PBMC, peripheral blood mononuclear cells; PCT, plateletcrit; PLT, platelet count; TJC, tender joint count; RA, rheumatoid arthritis; WBC, white blood cell count.

A

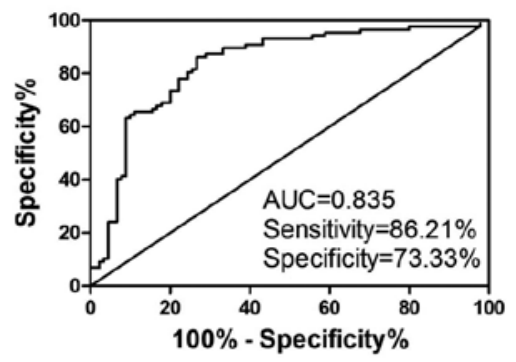

B

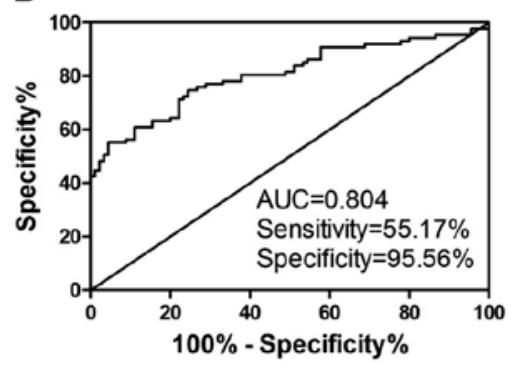

C

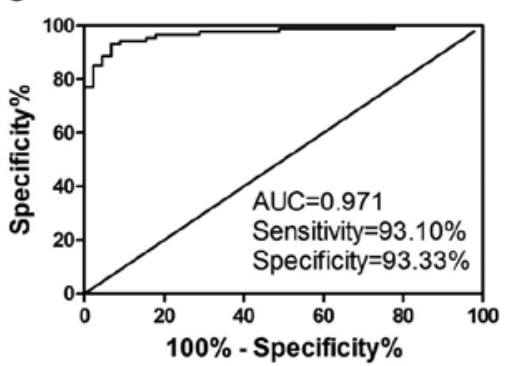

Figure 4. ROC curve analysis of PBMC hsa_circ_0000175 and hsa_circ_0008410 in RA patients. (A) ROC curve analysis of PBMC hsa_circ_0000175 in RA patients vs. HC. (B) ROC curve analysis of PBMC hsa_circ_0008410 in RA patients vs. HC. (C) ROC curve analysis of combined PBMC hsa_circ_0000175 and hsa_circ_0008410 in RA patients vs. HC. AUC, area under the curve; HC, healthy controls; PBMC, peripheral blood mononuclear cells; RA, rheumatoid arthritis; ROC, receiver operating characteristics.

PBMC hsa_circ_0000175 and hsa_circ_0008410 expression in RA, SLE and AS patients. As shown in Fig. 5A, there was significant differences between the expression of PBMC hsa_circ_0000175 in RA, SLE, and AS patients. The expression of PBMC hsa_circ_0000175 was significantly increased in RA patients compared with that in SLE patients, but markedly decreased compared with that in AS patients. In addition, the expression of PBMC hsa_circ_0008410 was markedly increased in RA patients compared with that in SLE and AS patients (Fig. 5B).

Next, ROC curves based on PBMC hsa_circ_0000175 and hsa_circ_0008410 were further analyzed in RA and SLE patients. The AUC for PBMC hsa_circ_0000175 in RA and SLE patients was 0.642 , with a sensitivity of $60.92 \%$ and a 

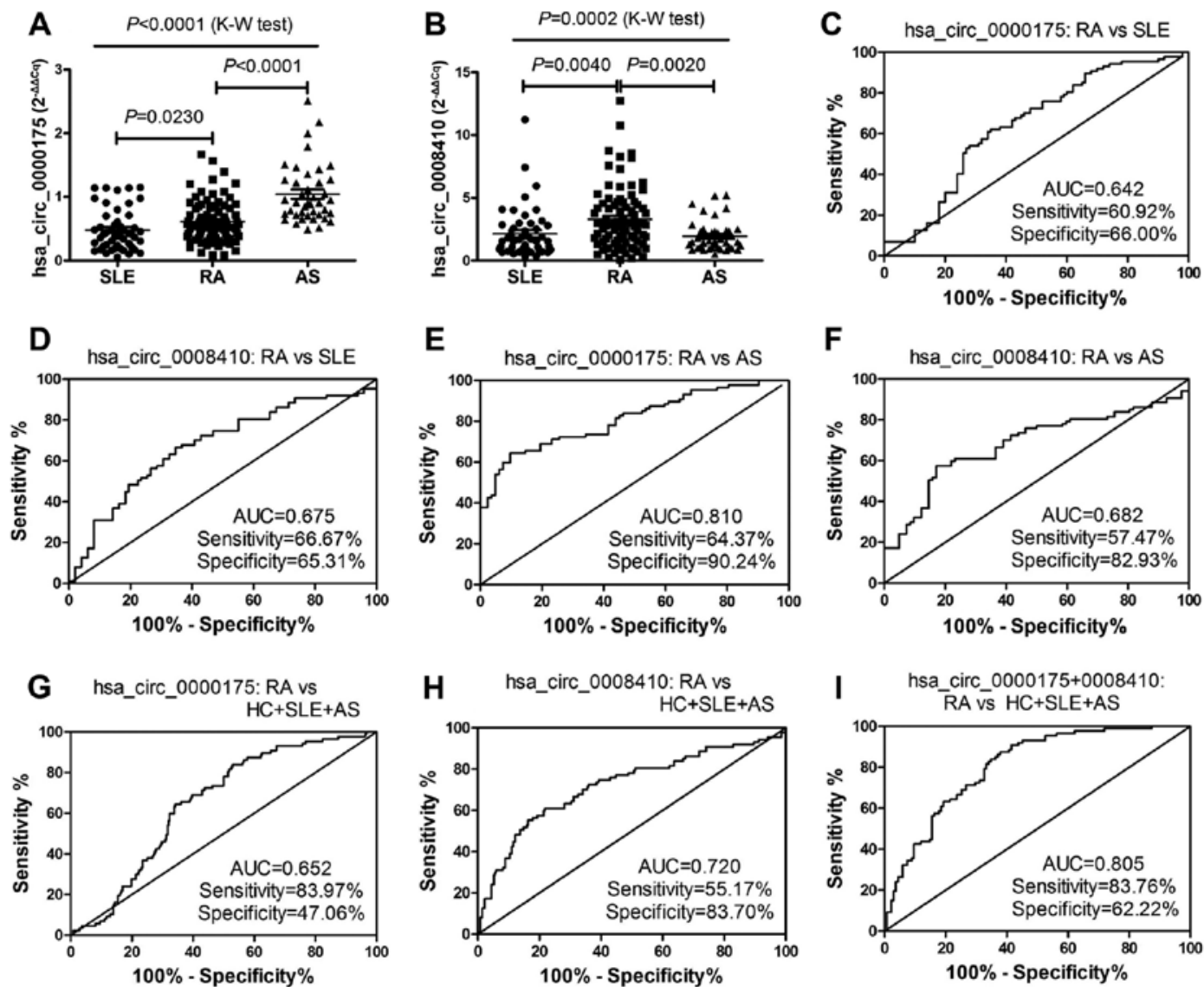

Figure 5. ROC curve analysis of the risk score of PBMC hsa_circ_0000175 and hsa_circ_0008410. (A) There was a significant difference between RA, SLE, and AS patients in the expression of PBMC hsa_circ_0000175 (Kruskal-Wallis test). The expression of PBMC hsa_circ_0000175 was markedly increased in RA compared with SLE patients (Dunn's post-hoc test), while the expression of PBMC hsa_circ_0000175 was markedly decreased in RA compared with AS patients (Dunn's post-hoc test). (B) There was a significant difference between RA, SLE, and AS patients in the expression of PBMC hsa_circ_0008410 (Kruskal-Wallis test). The expression of PBMC hsa_circ_0008410 was markedly increased in RA compared with SLE (Dunn's post-hoc test) and AS patients (Dunn's post-hoc test). (C) ROC curve analysis of PBMC hsa_circ_0000175 in RA vs. SLE patients. (D) ROC curve analysis of PBMC hsa_circ_0008410 in RA vs. SLE patients. (E) ROC curve analysis of PBMC hsa_circ_0000175 in RA vs. AS patients. (F) ROC curve analysis of PBMC hsa_circ_0008410 in RA vs. AS patients. (G) ROC curve analysis of PBMC hsa_circ_0000175 in RA patients vs. controls (HC + SLE + AS). (H) ROC curve analysis of PBMC hsa_circ_0008410 in RA patients vs. controls (HC + SLE + AS). (I) ROC curve analysis of combined PBMC hsa_circ_0000175 and hsa_circ_0008410 in RA patients vs. controls (HC + SLE + AS). AUC, area under the curve; AS, ankylosing spondylitis; HC, healthy controls; K-W test, Kruskal-Wallis test; PBMC, peripheral blood mononuclear cells; RA, rheumatoid arthritis; ROC, receiver operating characteristics; SLE, systemic lupus erythematosus.

specificity of $66.00 \%$ (Fig. 5C), and the AUC for PBMC hsa_circ_0008410 in RA and SLE patients was 0.675, with a sensitivity of $66.67 \%$ and a specificity of $65.31 \%$ (Fig. 5D). The expression of PBMC hsa_circ_0000175 and hsa_circ_0008410 were also successful in distinguishing RA from AS patients. The AUC for PBMC hsa_circ_0000175 in RA and AS patients was 0.810 , with a sensitivity of $64.37 \%$ and a specificity of 90.24\% (Fig. 5E), and the AUC for PBMC hsa_circ_0008410 in RA and AS patients was 0.682, with a sensitivity of $57.47 \%$ and a specificity of $82.93 \%$ (Fig. 5F). In addition, the expression of PBMC hsa_circ_0000175 and hsa_circ_0008410 distinguished RA patients from all controls $(\mathrm{HC}+\mathrm{SLE}+\mathrm{AS})$. The AUC for PBMC hsa_circ_0000175 in RA patients and all controls $(\mathrm{HC}+\mathrm{SLE}+\mathrm{AS})$ was 0.652 , with a sensitivity of $83.97 \%$ and a specificity of $47.06 \%$ (Fig. 5G), the AUC for PBMC hsa_circ_0008410 in RA patients and all controls $(\mathrm{HC}+\mathrm{SLE}+\mathrm{AS})$ was 0.720 , with a sensitivity of $55.17 \%$ and a specificity of $83.70 \%$ (Fig. $5 \mathrm{H}$ ), and the AUC for the combination of PBMC hsa_circ_0000175 and hsa_circ_0008410 in RA patients and all controls $(\mathrm{HC}+\mathrm{SLE}+\mathrm{AS})$ was 0.805 , with a sensitivity of $83.76 \%$ and a specificity of $62.22 \%$ (Fig. 5I).
Expression levels of PBMC hsa_circ_0000175 and hsa_ circ_0008410 are risk factors for $R A$. The aforementioned results demonstrated that the expression levels of PBMC hsa circ_0000175 and hsa_circ_0008410 in RA were different from HC, SLE and AS, and were associated with the activity and severity of RA. Thus, to investigate whether the expression labels of PBMC hsa_circ_0000175 and hsa_circ_0008410 were risk factors for RA, the 'enter method' of logistic regression was used. As shown in Table IV, the equation on the expression of hsa_circ_0000175 and hsa_circ_0008410 in PBMC was as follows: $\mathrm{Y}=-2.019 \mathrm{X} 1$ (hsa_circ_0000175) $+0.550 \mathrm{X} 2$ (hsa_circ_0008410) - 0.292. The expression levels of PBMC hsa_circ_0000175 and hsa_circ_0008410 were identified as risk factors for RA (all $\mathrm{P}<0.0001$ ).

hsa_circ_0000175/miRNA, hsa_circ_0008410/miRNA interaction analysis. To confirm the function of hsa circ_0000175 and hsa_circ_0008410, potential miRNA targets of the circRNAs were predicted by aligning with the miRNA response elements (MREs) using Arraystar's home-made miRNA target prediction software based on TargetScan (24) 
Table IV. Expression of PBMC hsa_circ_0000175 and hsa_circ_0008410 in equation.

\begin{tabular}{lccccrr}
\hline Item & B & S.E & Wald & df & P & Exp (B) \\
\hline hsa_circ_0000175 & -2.019 & 0.443 & 20.742 & 1 & $<0.0001$ & 0.133 \\
hsa_circ_0008410 & 0.550 & 0.106 & 26.877 & 1 & $<0.0001$ & 1.733 \\
Constant & -0.292 & 0.351 & 0.694 & 1 & 0.4050 & 0.747 \\
\hline
\end{tabular}

circRNAs, circular RNAs.

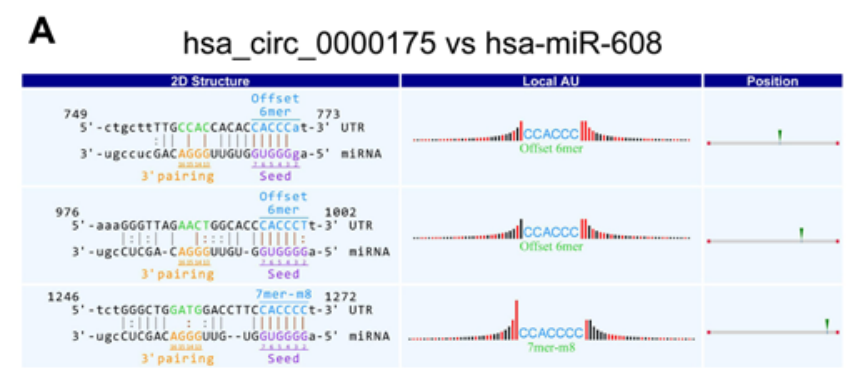

B hsa_circ_0008410 vs hsa-miR-6776-3p
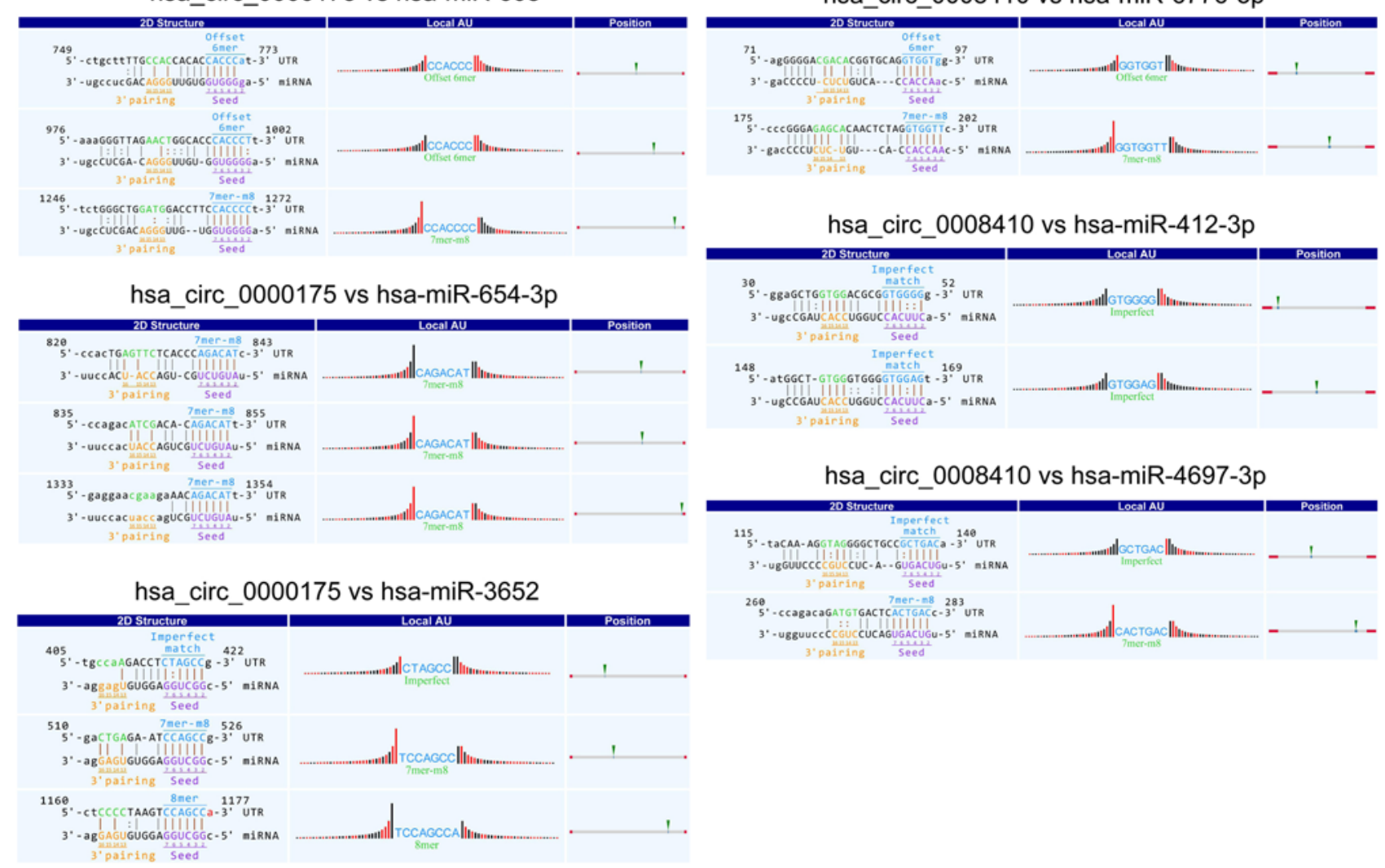

Figure 6. Snippet of the detailed annotation for circRNA/miRNA interactions. (A) hsa_circ_0000175/hsa-miR-608, hsa_circ_0000175/hsa-miR-654-3p and hsa_circ_0000175/hsa-miR-3652 interaction. (B) Hsa_circ_0008410/hsa-miR-6776-3p, hsa_circ_0008410/hsa-miR-412-3p and hsa_circ_0008410/ hsa-miR-4697-3p. CircRNA, circular RNA; miRNA, microRNA.

and miRanda (25). Three putative miRNA targets of hsa_circ_0000175 were identified, including hsa-miR-608, hsa-miR-654-3p and hsa-miR-3652. Three putative miRNA targets of hsa_circ_0008410 were also identified, including hsa-miR-6776-3p, hsa-miR-412-3p and hsa-miR-4697-3p. The molecular interactions between these two circRNAs and the target miRNAs mentioned above are illustrated in Fig. 6.

\section{Discussion}

It has been previously reported that PBMC circRNAs may be associated with RA. In 2017, Ouyang et al (17) and Zheng et al (26) simultaneously investigated differentially expressed circRNAs in PBMCs from RA patients by microarray and RT-qPCR. In addition, Tang et al (19) recently demonstrated that the expression of ciRS-7 was increased in PBMCs from RA patients. However, little was known regarding the expression of hsa_circ_0000175 and hsa_circ_0008410 in PBMCs from RA. The present study demonstrated that PBMC hsa_circ_0000175 and hsa_circ_0008410 were markedly decreased and increased, respectively, in RA patients compared with HC.

As shown in Table III, the clinical manifestations (DAS28, TJC, SJC and VAS) and laboratory indicators (autoantibodies, inflammation markers and immune cells) in RA were different from the controls, indicating the activity and severity of RA. Spearman's rho correlation analysis revealed that PBMC hsa_circ_0000175 was correlated with the ACPA titer, which is a biomarker of RA and reflects its activity (27). In addition, PBMC hsa_circ_0000175 was correlated with inflammatory markers, including WBC, N, N\%, L, L\% and NLR, which reflect the activity and severity of RA (28). Moreover, PBMC hsa_circ_0008410 was correlated with TJC, disease duration 
and PLT, which are associated with the development of peripheral neuropathy and are correlated with DAS28 (29,30), and PCT, which is associated with the treatment of RA (31). Thus, these data indicated that PBMC hsa_circ_0000175 and hsa_ circ_0008410 were associated with the activity and severity of RA.Importantly, logistic regression analysis revealed that PBMC hsa_circ_0000175 and hsa_circ_0008410 were risk factors for RA, suggesting that hsa_circ_0000175 and hsa_circ_0008410 may be involved in the pathogenesis of RA.

Recent evidence has indicated that circRNAs may serve as novel biomarkers in the diagnosis of RA (17-19). To explore whether PBMC hsa_circ_0000175 and hsa_circ_0008410 constitute biomarkers for the diagnosis of RA, they were analyzed in larger patient cohorts using ROC curves. The cut-off values of hsa_circ_0000175 and hsa_circ_0008410 that best distinguished RA patients from $\mathrm{HC}$ were determined. Hsa_circ_0000175 had an AUC of 0.835, a specificity of $73.33 \%$ and a sensitivity of $86.21 \%$ for RA, whereas hsa_circ_0008410 had an AUC of 0.804 , a specificity of $95.56 \%$ and a sensitivity of $55.17 \%$. The logistic regression model using both targets in combination exhibited an improved ability for distinguishing RA patients from HC, with an AUC of 0.971, a sensitivity of $93.10 \%$ and a specificity of $93.33 \%$, indicating the additive effects of the two circRNAs in terms of diagnostic value. The diagnostic potential appears to be superior to that of blood biomarkers of RA reported previously, particularly in terms of AUC and specificity (17-19). Subsequently, the ability of PBMC hsa_circ_0000175 and hsa_circ_0008410 to effectively distinguish RA from other autoimmune diseases, such as SLE and AS, was assessed. As aforementioned, PBMC hsa_circ_0000175 and hsa_circ_0008410 were successful in distinguishing RA patients from SLE patients, AS patients and HC.

It is well-known that circRNAs may act as miRNA sponges and regulate target genes to alter the course of disease development. Li et al (32) reported that hsa_circ_0001859 regulated ATF2 expression by acting as an miR-204/211 sponge in human RA. Our previous study (18) demonstrated that hsa-miR-892a, an miRNA target of hsa_circ_0044235, was increased in RA patients, while hsa_circ_0044235 was decreased,indicating that hsa_circ_0044235 may play a role in RA by interacting with hsa-miR-892a. Multiple miRNAs are implicated in the occurrence and development of RA $(33,34)$. Bioinformatics predicted that hsa-miR-608, hsa-miR-654-3p and hsa-miR-3652 may be potential targets of hsa_circ_0000175. Hsa_circ_0008410 was shown to potentially bind hsa-miR-6776-3p, hsa-miR-412-3p and hsa-miR-4697-3p. Although most potential miRNAs interacting with circRNAs have been predicted for RA, no study on the function of these miRNAs in RA has been published to date, to the best of our knowledge.

However, several limitations of the present study should be acknowledged. Firstly, six circRNAs that were differentially expressed in both PBMC from SLE patients in our previous study and $\mathrm{T}$ cells from SLE patients in previous literature were selected, and the possibility of using them as biomarkers for diagnosis of RA was explored. Although RA and SLE exhibit different pathogenies, these two diseases have similar pathological and immunological abnormalities. Furthermore, there were significant differences in the levels of PBMC hsa_circ_0000175 and hsa_circ_0008410 between RA and SLE patients. Thus, the possibility of hsa_circ_0000175 and hsa_circ_0008410 being used as biomarkers for diagnosis of RA require further exploration. Secondly, the sample size of the patients with new-onset RA, SLE, AS and HC was relatively small, and the samples were sourced from only one hospital, which may limit the universality of the results. Therefore, the current findings require confirmation in larger and more diverse samples.

In conclusion, the PBMC hsa_circ_0000175, hsa_ circ_0008410, and combination of hsa_circ_0000175 and hsa_circ_0008410, may improve the diagnostic accuracy for RA. In addition, the expression levels of PBMC hsa_circ_0000175 and hsa_circ_0008410 were found to be associated with the activity and severity of RA.

\section{Acknowledgements}

The authors would like to acknowledge Dr Rui Wu (Department of Rheumatology, the First Affiliated Hospital of Nanchang University, Jiangxi, China) for their help in screening patients and collecting specimens.

\section{Funding}

The present was supported by the Key Research and Development Plan Project of Jiangxi Province (grant no. 20181BBG70013), the Science and Technology Plan Project of the Education Department of Jiangxi Province (grant no. 170008), the National Natural Science Foundation of China (grant nos. 81360459 and 81660277), the Jiangxi Provincial Natural Science Foundation of China (grant nos. 20151BAB215031 and 20171BAB205113), the Science and Technology Project of Health and Family Planning Commission of Jiangxi Province of China (grant no. 20165094) and the Foundation for Distinguished Young Scientists of Jiangxi Province of China (grant no. 20171BCB23087).

\section{Availability of data and materials}

The data used and/or analyzed during the current study are available from the corresponding author on reasonable request.

\section{Authors' contributions}

QL, LLZ and LBZ performed the experiments. JYR, LZ, YG, ZKH and JML analyzed and interpreted the data. QL and JML made substantial contributions to the design and supervision of the present study, and wrote the manuscript. All authors have reviewed the results and approved the final version of the manuscript.

\section{Ethics approval and consent to participate}

The present study was approved by the Ethics Committee of the First Affiliated Hospital of Nanchang University. All participants provided informed consent prior to inclusion in the study.

\section{Patient consent for publication}

Not applicable. 


\section{Competing interests}

The sauthors declare that they have no competing interests.

\section{References}

1. McInnes IB and Schett G: Cytokines in the pathogenesis of rheumatoid arthritis. Nat Rev Immunol 7: 429-442, 2007.

2. Aletaha D, Neogi T, Silman AJ, Funovits J, Felson DT, Bingham CO III, Birnbaum NS, Burmester GR, Bykerk VP, Cohen MD, et al: 2010 rheumatoid arthritis classification criteria: An American College of Rheumatology/European League Against Rheumatism collaborative initiative. Ann Rheum Dis 69: 1580-1588, 2010.

3. Cohen S and Emery P: The American College of Rheumatology/European League Against Rheumatism criteria for the classification of rheumatoid arthritis: A game changer. Arthritis Rheum 62: 2592-2594, 2010.

4. Abdul-Maksoud RS, Sediq AM, Kattaia A, Elsayed W, Ezzeldin N, Abdel Galil SM and Ibrahem RA: Serum miR-210 and miR-155 expression levels as novel biomarkers for rheumatoid arthritis diagnosis. Br J Biomed Sci 7: 1-5, 2017.

5. Wang W, Zhang Y, Zhu B, Duan T, Xu Q, Wang R, Lu L and Jiao Z: Plasma microRNA expression profiles in Chinese patients with rheumatoid arthritis. Oncotarget 6: 42557-42568, 2015.

6. Lu MC, Yu HC, Yu CL, Huang HB, Koo M, Tung CH and Lai NS: Increased expression of long noncoding RNAs LOC100652951 and LOC100506036 in T cells from patients with rheumatoid arthritis facilitates the inflammatory responses. Immunol Res 64: 576-583, 2016

7. Zhang HJ, Wei QF, Wang SJ, Zhang HJ, Zhang XY, Geng Q, Cui YH and Wang XH: LncRNA HOTAIR alleviates rheumatoid arthritis by targeting miR-138 and inactivating NF- $\kappa \mathrm{B}$ pathway. Int Immunopharmacol 50: 283-290, 2017.

8. Qu S, Yang X, Li X, Wang J, Gao Y, Shang R, Sun W, Dou K and Li H: Circular RNA: A new star of noncoding RNAs. Cancer Lett 365: 141-148, 2015 .

9. Chen LL and Yang L: Regulation of circRNA biogenesis. RNA Biol 12: 381-388, 2015.

10. Fischer JW and Leung AK: CircRNAs: A regulator of cellular stress. Crit Rev Biochem Mol Biol 52: 220-233, 2017.

11. Wu HJ, Zhang CY, Zhang S, Chang M and Wang HY: Microarray expression profile of circular RNAs in heart tissue of mice with myocardial infarction-induced heart failure. Cell Physiol Biochem 39: 205-216, 2016.

12. Kulcheski FR, Christoff AP and Margis R: Circular RNAs are miRNA sponges and can be used as a new class of biomarker. J Biotechnol 238: 42-51, 2016.

13. Salzman J, Chen RE, Olsen MN, Wang PL and Brown PO: Cell-type specific features of circular RNA expression. PLoS Genet 9: e1003777, 2013.

14. Rybak-Wolf A, Stottmeister C, Glazar P, Jens M, Pino N, Giusti S, Hanan M, Behm M, Bartok O, Ashwal-Fluss R, et al: Circular RNAs in the mammalian brain are highly abundant, conserved, and dynamically expressed. Mol Cell 58: 870-885, 2015.

15. Huang Z, Su R, Qing C, Peng Y, Luo Q and Li J: Plasma circular RNAs hsa_circ_0001953 and hsa_circ_0009024 as diagnostic biomarkers for active tuberculosis. Front Microbiol 9: 2010, 2018.

16. Bach DH, Lee SK and Sood AK: Circular RNAs in cancer. Mol Ther Nucleic Acids 16: 118-129, 2019.

17. Ouyang Q, Wu J, Jiang Z, Zhao J, Wang R, Lou A, Zhu D, Shi GP and Yang M: Microarray expression profile of circular RNAs in peripheral blood mononuclear cells from rheumatoid arthritis patients. Cell Physiol Biochem 42: 651-659, 2017.
18. Luo Q, Zhang L, Li X, Fu B, Deng Z, Qing C, Su R, Xu J, Guo Y, Huang Z and Li J: Identification of circular RNAs hsa_circ 0044235 in peripheral blood as novel biomarkers for rheumatoid arthritis. Clin Exp Immunol 194: 118-124, 2018.

19. Tang X, Wang J, Xia X, Tian J, Rui K, Xu H and Wang S: Elevated expression of ciRS-7 in peripheral blood mononuclear cells from rheumatoid arthritis patients. Diagn Pathol 14: 11, 2019.

20. Luo Q,Zhang L, Li X, Fu B, Guo Y,Huang Z and Li J: Identification of circular RNAs hsa_circ_0044235 and hsa_circ_0068367 as novel biomarkers for systemic lupus erythematosus. Int J Mol Med 44: 1462-1472, 2019.

21. Li LJ, Zhu ZW, Zhao W, Tao SS, Li BZ, Xu SZ, Wang JB, Zhang MY, Wu J, Leng RX, et al: Circular RNA expression profile and potential function of hsa_circ_0045272 in systemic lupus erythematosus. Immunology 155: 137-149, 2018.

22. Hochberg MC: Updating the American College of Rheumatology revised criteria for the classification of systemic lupus erythematosus. Arthritis Rheum 40: 1725, 1997.

23. van der Linden S, Valkenburg HA and Cats A: Evaluation of diagnostic criteria for ankylosing spondylitis. A proposal for modification of the New York criteria. Arthritis Rheum 27: 361-368, 1984.

24. Enright A, John B, Gaul U, Tuschl T, Sander C and Marks DS: MicroRNA targets in Drosophila. Genome Biol 5: R1, 2003.

25. Pasquinelli AE: MicroRNAs and their targets: Recognition, regulation and an emerging reciprocal relationship. Nat Rev Genet 13: 271-282, 2012.

26. Zheng F, Yu X, Huang J and Dai Y: Circular RNA expression profiles of peripheral blood mononuclear cells in rheumatoid arthritis patients, based on microarray chip technology. Mol Med Rep 16: 8029-8036, 2017.

27. Hafkenscheid L, de Moel E, Smolik I, Tanner S, Meng X, Jansen BC, Bondt A, Wuhrer M, Huizinga TWJ, Toes REM, et al: $\mathrm{N}$-linked glycans in the variable domain of ACPA-IgG predict the development of rheumatoid arthritis. Arthritis Rheumatol 71: 1626-1633, 2019.

28. Sargin G, Senturk T, Yavasoglu I and Kose R: Relationship between neutrophil-lymphocyte, platelet-lymphocyte ratio and disease activity in rheumatoid arthritis treated with rituximab. Int J Rheum Dis 21: 2122-2127, 2018.

29. Li Y, Jiang L, Zhang Z, Li H, Jiang L, Wang L and Li Z: Clinical characteristics of rheumatoid arthritis patients with peripheral neuropathy and potential related risk factors. Clin Rheumatol 38: 2099-2107, 2019.

30. Zhou L, Xiao DM, Qin W, Xie BH, Wang TH, Huang H, Zhao BJ, Han X, Sun QQ, Wu XD and Cen H: The clinical value of hematological markers in rheumatoid arthritis patients treated with tocilizumab. J Clin Lab Anal 19: e22862, 2019.

31. Liu J, Cao Y, Huang C, Wang Y, Chen X, Zhang W, Wang G, Fan H, Ge Y, Chen R, et al: Use of xinfeng capsule to treat abarticular pathologic changes in patients with rheumatoid arthritis. J Tradit Chin Med 34: 532-538, 2014.

32. Li B, Li N, Zhang L, Li K, Xie Y, Xue M and Zheng Z: Hsa circ 0001859 regulates ATF2 expression by functioning as an MiR-204/211 sponge in human Rheumatoid Arthritis. J Immunol Res 2018: 9412387, 2018.

33. Qu Y, Zhang YP, Wu J, Jie LG, Deng JX, Zhao DB and Yu QH: Downregulated microRNA-135a ameliorates rheumatoid arthritis by inactivation of the phosphatidylinositol 3-kinase/AKT signaling pathway via phosphatidylinositol 3-kinase regulatory subunit 2. J Cell Physiol 234: 17663-17676, 2019.

34. Philippe L, Alsaleh G, Bahram S, Pfeffer S and Georgel P: The miR-17 92 Cluster: A key player in the control of inflammation during Rheumatoid Arthritis. Front Immunol 4: 70, 2013. 